Progress in Flight Physics 5 (2013) 209-234

DOI: $10.1051 /$ eucass/201305209

(C) Owned by the authors, published by EDP Sciences, 2013

\title{
INVESTIGATIONS ON THE TURBULENT WAKE OF A GENERIC SPACE LAUNCHER GEOMETRY IN THE HYPERSONIC FLOW REGIME
}

\author{
D. Saile ${ }^{1}$, A. Gülhan ${ }^{1}$, A. Henckels ${ }^{1}$, C. Glatzer ${ }^{2}$, \\ V. Statnikov ${ }^{2}$, and M. Meinke ${ }^{2}$ \\ ${ }^{1}$ Institute of Aerodynamics and Flow Technology \\ German Aerospace Center \\ Linder Höhe, Köln 51147, Germany \\ ${ }^{2}$ Institute of Aerodynamics Aachen \\ RWTH Aachen University \\ 5a Wüllnerstraße, Aachen 52062, Germany
}

The turbulent wake flow of generic rocket configurations is investigated experimentally and numerically at a freestream Mach number of 6.0 and a unit Reynolds number of $10 \cdot 10^{6} \mathrm{~m}^{-1}$. The flow condition is based on the trajectory of Ariane V-like launcher at an altitude of $50 \mathrm{~km}$, which is used as the baseline to address the overarching tasks of wake flows in the hypersonic regime like fluid-structural coupling, reverse hot jets and base heating. Experimental results using pressure transducers and the high-speed Schlieren measurement technique are shown to gain insight into the local pressure fluctuations on the base and the oscillations of the recompression shock. This experimental configuration features a wedgeprofiled strut orthogonally mounted to the main body. Additionally, the influence of cylindrical dummy nozzles attached to the base of the rocket is investigated, which is the link to the numerical investigations. Here, the axisymmetric model possesses a cylindrical sting support of the same diameter as the dummy nozzles. The sting support allows investigations for an undisturbed wake flow. A time-accurate zonal Reynolds-Averaged Navier-Stokes / Large Eddy Simulation (RANS/LES) approach is applied to identify shocks, expansion waves, and the highly unsteady recompression region numerically. Subsequently, experimental and numerical results in the strut-averted region are compared with regard to the wall pressure and recompression shock frequency spectra. For the compared configurations, experimental pressure spectra exhibit dominant Strouhal numbers at about $\mathrm{Sr}_{D}=0.03$ and 0.27 , and the recompression shock oscillates at 0.2 . In general, the pressure and recompression shock fluctuations numerically calculated agree reasonably with the experimental results. The experiments with a blunt base reveal base-pressure spectra with dominant Strouhal numbers at 0.08 at the center position and $0.145,0.21-0.22$, and $0.31-0.33$ at the outskirts of the base. 


\section{INTRODUCTION}

At high altitudes, launch vehicles face various tasks. The unsteady recirculation zone features periodic pressure fluctuations that can lead to dynamic loads on the rocket structure. The aft section of the rocket is also exposed to reverse hot jets resulting from the transport of hot gas from the nozzle plume upstream to the base. This can cause confined hot spots and also increases the overall thermal load on the components in the region, e.g., the nozzle external surface and the vehicle base. Further, for supersonic projectiles, Rollstin [1] determines the base drag caused by the separation of the outer flow to be up to $35 \%$ of the overall drag, which could be even higher in the case of launch vehicles having a larger base area and might excite vibrations of critical amplitude.

These considerations drive the interest to expand the understanding of the base flow physics in the hypersonic regime and offer a strong motivation of wake flow investigations. In spite of mostly simple base geometries, the flow field is highly complex, covering the interaction of the recirculation region with the separated shear layer, expansion waves and a recompression region. These aerodynamic characteristics affect the overall axial force and exhibit periodic and stochastic flow phenomena. Therefore, it is of high interest to provide accurate analyses of the not yet fully understood time-dependent base flow to design reliable future space systems. In the recent years, the extensive experimental $[2,3]$ and numerical [4-7] work was executed with focus on unsteady effects.

A sketch of the mean flow features is given in Fig. 1. The icon above shows the region of interest (ROI) with reference to the overall wind tunnel model. The incoming supersonic flow and the boundary layer on the surface of the model

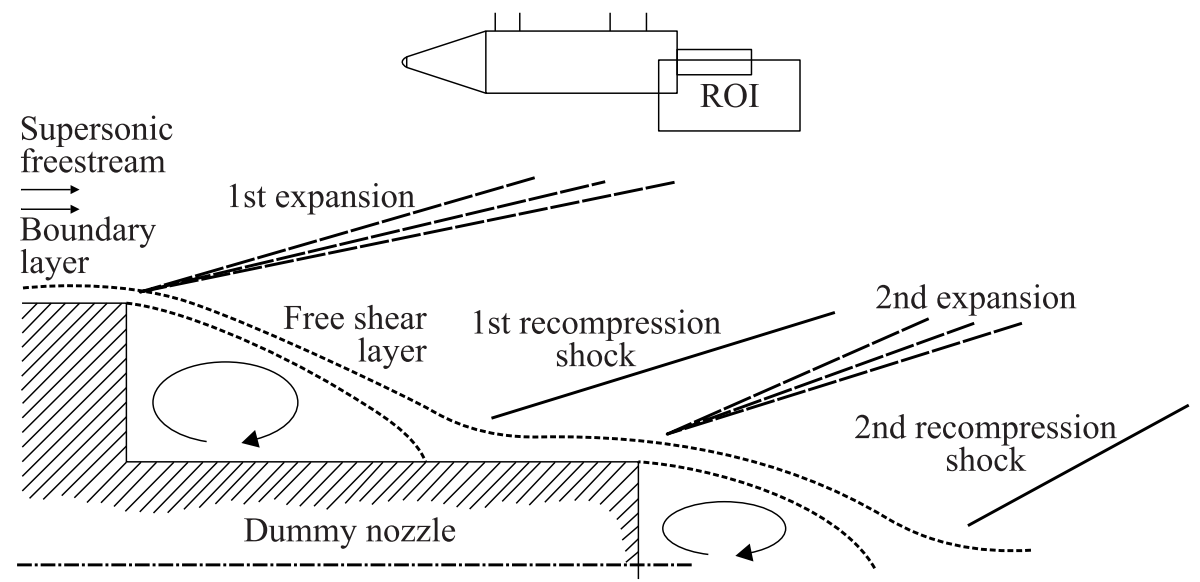

Figure 1 Schematics of the mean base flow features 


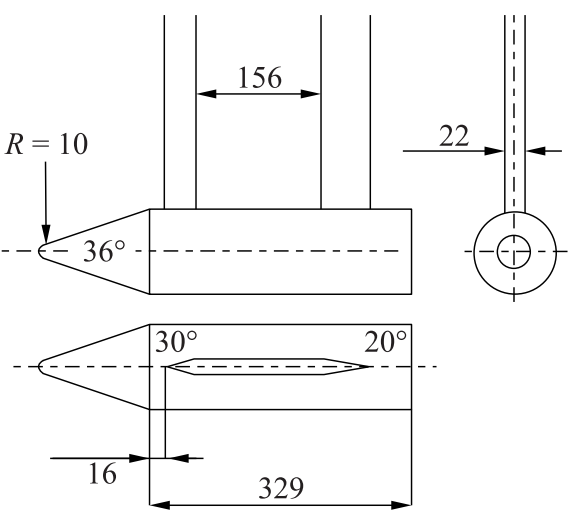

(a)

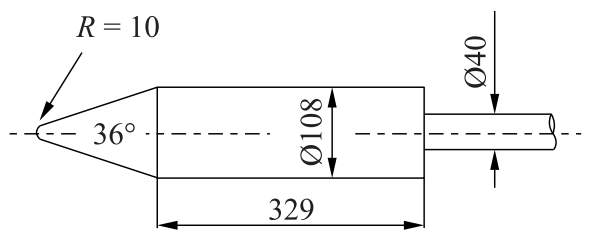

(b)

Figure 2 Geometries of the generic rocket configuration with strut support: $(a)$ configuration $A$ and $(b)$ configuration $B$. Dimensions are in millimeters

experience an abrupt geometry change at the end of the model, which results in a flow separation. The separated flow expands, meaning a deflection towards the axis takes place. A high energetic free shear layer evolves right from the edge of the base, which separates the inviscid external flow from the recirculation region downstream of the base. The shear layer is subjected to an adverse pressure gradient before reattaching on the solid surface, whereas the flow consequently realigns along the nozzle in the axial direction. This is where compression waves emanate and focus to a recompression shock.

The exemplary configuration with a dummy nozzle exhibits a second stage of the expansion and the recompression at the rear end of the nozzle. Downstream of the dummy nozzle, a free shear layer reattachment takes place, which essentially can also be found when no nozzle is attached to the base. No second expansion and recompression occur in the current simulation since the numerically investigated configuration is held by a sting at a constant diameter equal to the generic nozzle dummy.

The investigated models are representations of the Ariane V configuration with respect to the main geometrical bodies. The experiments and numerical simulations are carried out on identical generic models that only differ by the support in the flow field. Figure $2 a$ shows a sketch of the experimental model, called configuration $A$, which is supported by a strut, and Fig. $2 b$ shows configuration $B$ for the numerical investigations, which is supported by a sting mounted at the base of the main body. This support sting mimics the contour of a nozzle and allows for investigations of an axially undisturbed base flow in order to evaluate the influence of the strut support.

The same flow topology can also be found with a jet flow through the nozzle if the exterior flow attaches completely along the nozzle surface. A shortening 
of the nozzle results at one point in a non-attached exterior flow due to the displacement effect caused by the jet flow. In this case, the recirculation region is enclosed by the shear layer of the exterior and the jet flow. Both flows consequently have an influence on the recirculation region.

As the baseline for the investigations, an assumed trajectory stage of an Ariane V-like space launcher [8] is defined simulating the launch conditions at an altitude of $50 \mathrm{~km}$, which corresponds to a freestream Mach number of 6.0 and a unit Reynolds number of $10 \cdot 10^{6} \mathrm{~m}^{-1}$. In this flow regime, the fluid-structure interaction is less significant, while unsteady shock phenomena in the base region become more important.

The objective of the present paper is experimental and numerical investigation of the turbulent wake flow with focus on unsteady flow behavior by means of base-pressure fluctuations and fluctuations of the recompression shock. The advantages of both methods of investigations are exploited, and experimental and numerical results are compared to each other. The experimental side makes use of the flexibility to investigate the behavior of the wake to different dummy nozzles, whereas the numerical simulations provides detailed time-resolved data about all flow parameters in the field of interest. Another complementing aspect of the numerical investigations concerns the influence of the strut. Ottens et al. [9] showed that the base-pressure decreases considerably due to a wind tunnel strut. For the numerical investigations, the axisymmetric model possesses a cylindrical sting support, which allows investigations for a wake flow being not asymmetrically disturbed by the effects of a vertical wind tunnel strut used for the configuration with a nozzle dummy.

The experiments are conducted with Kulite pressure transducers and the high-speed Schlieren measurement technique. For the numerical simulations, a zonal RANS/LES approach is applied. The RANS models are not capable of predicting accurate unsteady data and also fail to provide accurate results concerning the low pressure recirculation area behind the base, while the predictions of the attached flow around the main body are quite satisfactory. At present, direct numerical simulation (DNS) is restricted to small Reynolds numbers and a small integration domain. Hence, RANS simulations are used to predict the attached main body flow field while LES computations are applied to the unsteady wake flow using the RANS results as inflow conditions. The turbulent viscosity of the RANS model is used to generate physical turbulent fluctuations at the inlet of the LES domain.

\section{METHODS}

As mentioned in the introduction, the experimental and numerical investigations are executed on comparable models. The exterior geometry is an assembly of simple geometrical bodies. It consists of a spherical nose with a radius of $10 \mathrm{~mm}$ that 
is attached to the cylindrical main body with a diameter of $D=108 \mathrm{~mm}$ and a length of $328.6 \mathrm{~mm}$ via the cone with an apex angle of $36^{\circ}$.

In Fig. 3, the experimental wind tunnel equipped with a strut is shown. The strut is defined by a front wedge with an apex angle of $30^{\circ}$, a shaft with a width of $22 \mathrm{~mm}$ and a length of $156 \mathrm{~mm}$ and rear wedge with an opening angle of $20^{\circ}$. The

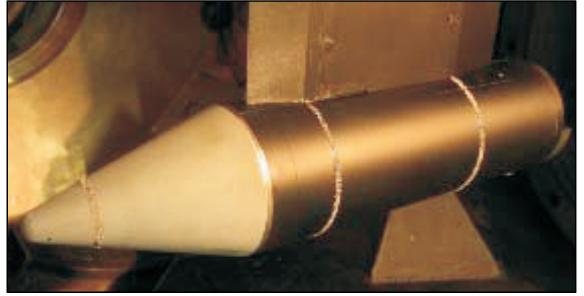

Figure 3 Wind tunnel model. reference configuration $A$ is modified during the experiments by mounting cylinders at the base to mimic the exterior geometry of a nozzle. No nozzle flow is applied. Nozzle lengths of $L / D=0$, $0.5,1.0$, and 1.2 are investigated. The diameter of the dummy nozzle for all experiments is kept at $d / D=0.4$, which corresponds to the diameter of the sting support for configuration $B$. Comparisons can be drawn to the measurements with configuration $A$ with the attached dummy nozzle.

Identical ambient flow parameters are defined for experiments and computations. A reservoir pressure of 10 bar and a reservoir temperature of $430 \mathrm{~K}$ at a freestream Mach number of 6.0 is applied, which equals a unit Reynolds number of $10 \cdot 10^{6} \mathrm{~m}^{-1}$ and to the ambient flow velocity of $871 \mathrm{~m} / \mathrm{s}$.

\subsection{Experimental Methods}

A detailed overview to the methods applied is described in [10]. Most of the measurement methods are identical. The measurements are performed in the supersonic wind tunnel $\mathrm{H} 2 \mathrm{~K}$ at the Institute of Aerodynamics and Flow Technology in the Supersonic and Hypersonic Technology Department located in Cologne. The wind tunnel H2K is a blow-down type wind tunnel equipped with a vacuum sphere to reach high Mach numbers. Six contoured, axially symmetrical aerodynamic testing nozzles are available for discrete Mach numbers within Mach 4.8 and 11.2. The exit diameter of the nozzle is $600 \mathrm{~mm}$. An electric heater with a maximum power of $5 \mathrm{MW}$ can heat the air in the reservoir chamber up to $1100 \mathrm{~K}$, which is necessary to omit condensation at high Mach numbers and can also be used to adjust the Reynolds number. A maximum unit Reynolds number of about $20 \cdot 10^{6} \mathrm{~m}^{-1}$ is reached at Mach 6 at a mass flow rate of almost $20 \mathrm{~kg} / \mathrm{s}$.

In order to trigger the transition of the boundary layer to a turbulent state, four tripping configurations were applied successively using carborundum with a grain size of $400 \mu \mathrm{m}$ and a zigzag-tape of the same height as tripping elements. First, a ring of carborundum with a width of $5 \mathrm{~mm}$ was applied on the nose at 
$45.4 \mathrm{~mm}$ (configuration 1) measured from the tip of the nose along the symmetry axis. Then, a second ring was added at $201.4 \mathrm{~mm}$ (configuration 2) on the cylindrical main body. Later, another ring was added at $352.4 \mathrm{~mm}$ (configuration 3 ). At last, the two rings on the main body were removed and replaced by a zigzag-tape at $201.4 \mathrm{~mm}$ (configuration 4). After having made the experiments that have shown the tripping elements have little influence on the basepressure behavior, the experiments presented here show the results with tripping configurations 3 and 4.

Steady and unsteady measurement techniques are applied. For the measurements concerning the boundary layer, the Pitot pressure is measured with a Pitot rake mounted at the end of the blunt base (Fig. 4). In order to incorporate the full boundary layer thickness, the rake

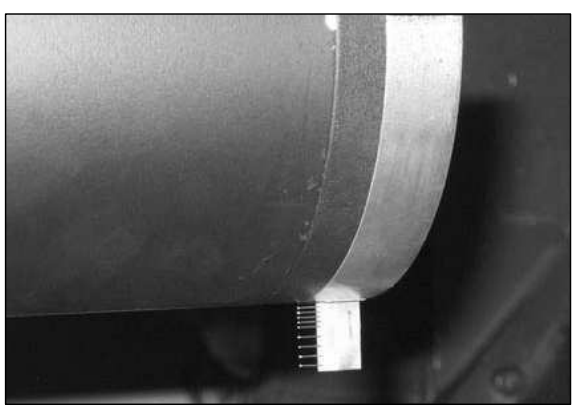

Figure 4 Pitot rake mounted on afterbody is elevated in a second run. Each pressure tube is connected to a 15 psi pressure scanner of Esterline Pressure Systems containing piezoresistive sensors and read with a system 8400 pressure data acquisition unit. The measurement uncertainties are $0.1 \%$ of full scale. The Pitot pressure is compared to results from RANS simulations on a two dimensional axisymmetric grid. The calculations are executed without (laminar) and with two different turbulence models (Spalart-Allmaras and Wilcox $k-\omega$ ) to reveal sensitivities.

In order to measure the unsteady behavior of the recompression shock behind the base, high-speed Schlieren technique is applied using an Ultima APX-RS camera of Photron. It is equipped with a 10 bit CMOS sensor, which provides a resolution of $1024 \times 1024$ pixel at a frame rate of $3000 \mathrm{fps}$ (frame per seconds) and a maximum frame rate of $250,000 \mathrm{fps}$ at a reduced resolution. The field of view is successively decreased to achieve high frame rates. Thus, the recompression shock is recorded with 10,000,16,800,20,000, and 22,500 fps, which results in 21,000 to 56,000 frames for spectral analysis. The number of frames is dependent on the resolution and limited by the internal memory of the highspeed camera. High-frequency pressure measurements are obtained with Kulite differential XCQ-080 pressure transducers, which feature typically a combined nonlinearity, hysteresis, and repeatability of $0.1 \%$. Six pressure transducers with a pressure range of 0.35 bar are flush mounted in plugs of the base (Fig. 5). The pressure reference tubes are connected to the ambient pressure of the measurement chamber. A glioblastoma multiforme (GBM) Viper system is used for the data acquisition purposes. The data is acquired over $10 \mathrm{~s}$ with a sampling rate of $96,000 \mathrm{~Hz}$. The cutoff frequency is set internally by the data acquisition system to $48,000 \mathrm{~Hz}$. 
Additionally, infrared images are taken using the ThermaCAM SC3000 NTS camera of FLIR (forward looking infrared) to gain information about the location of the boundary layer transition. These infrared images show only qualitative results, which refers to the fact that the surface material of the wind tunnel model is black-painted steel, meaning that the wall material features a high temperature conductivity and an unknown emission coefficient. For this reason, the heat flux can not be determined using infrared images only and fine structures smooth out. But the qualitative temperature distribution reveals the transition location, and additionally, gives insights to the disturbance effects

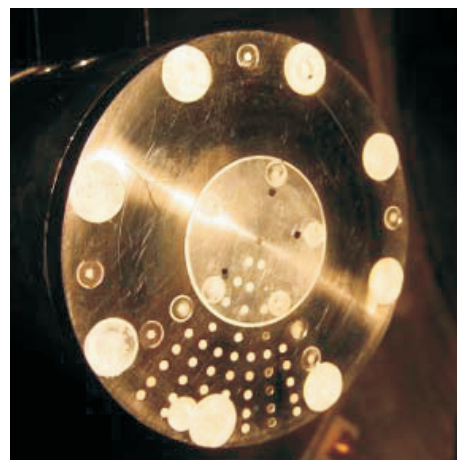

Figure 5 Instrumentation of base with Kulites pressure transducers. coming from the strut holding the model.

The Kulite pressure transducers are flush-mounted on the base in two different transducer arrangements. Figure $6 a$ shows the radial arrangement with the transducers on the opposite side of the strut $\left(\Phi=180^{\circ}\right)$ at a radius $r=0,12$, $25,35,45$, and $50 \mathrm{~mm}$. Further measurements are conducted with an angle of $\Phi=225^{\circ}, 270^{\circ}$, and $315^{\circ}$. Figure $6 b$ displays the azimuthal arrangement where the transducers are distributed angularly at a radial distance of $r=40 \mathrm{~mm}$ at $\Phi=170^{\circ}, 180^{\circ}, 190^{\circ}, 210^{\circ}$, and $240^{\circ}$. Additionally, another transducer is placed at $r=30 \mathrm{~mm}$ and $\Phi=180^{\circ}$.

The unsteady measurements from the high-speed camera and the pressure transducers are both analyzed using the fast Fourier transform (FFT) algorithm. The spectra are given as Strouhal number, which is defined as $\operatorname{Sr}_{D}=f D / u_{\infty}$ with the frequency $f$, the diameter of the base $D$, and the free stream velocity $u_{\infty}$.

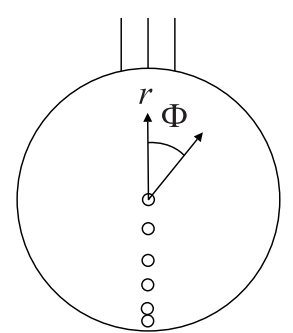

(a)

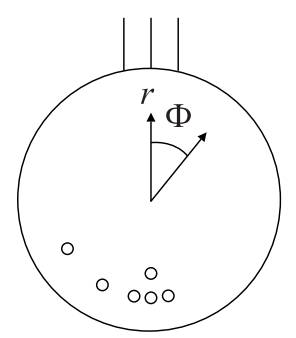

(b)

Figure 6 Radial $(a)$ and reference $(b)$ pressure transducer arrangements 


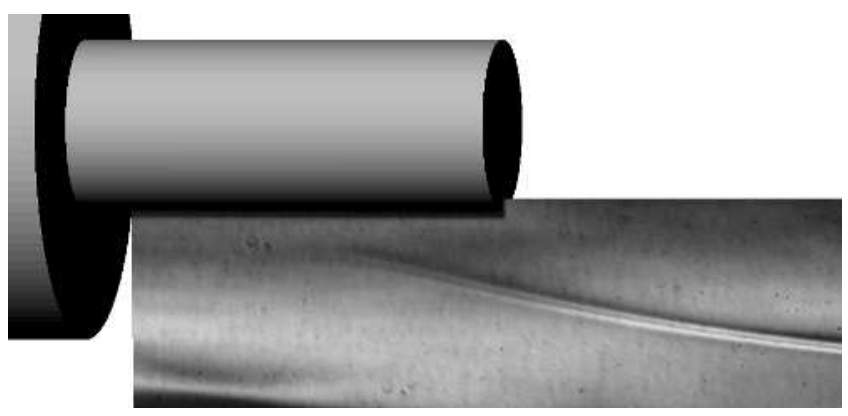

Figure 7 Standard deviation of a sequence of high-speed Schlieren measurement showing the recompression shock exemplary for a nozzle length of $L / D=1.2$ and a diameter of $d / D=0.4$

In the case of the high-speed Schlieren measurements, the vertical position of the shock is gained by analyzing the intensity profile at a defined location for each image. Then, the data sequence is segmented into sets of 1024 locations per frame with an overlap of $50 \%$ and multiplied with a Flattop window to suppress side-lobe leakage. Given by the number of samples mentioned above to resolve at least one wavelength and the Nyquist-Shannon sampling theorem, the frequency range that is covered with a recording of $10000 \mathrm{~Hz}$ is between $f_{\min }=0.18 \mathrm{~Hz}$ $\left(\mathrm{Sr}_{D \text { min }}=2.2 \cdot 10^{-} 5\right)$ and $f_{\max }=5000 \mathrm{~Hz}\left(\mathrm{Sr}_{D \text { max }}=0.6\right)$, respectively. For a recording frequency of $22500 \mathrm{~Hz}$, the minimum and maximum frequency is $f_{\min }=1 \mathrm{~Hz}\left(\mathrm{Sr}_{D \text { min }}=1.2 \cdot 10^{-4}\right)$ and $f_{\max }=11250 \mathrm{~Hz}\left(\mathrm{Sr}_{D \text { min }}=1.4\right)$. For the pressure transducers, segments of 8192 samples with an overlap of $50 \%$ are used in combination with a Hanning window. In the case with 960,000 samples at a sampling rate $96,000 \mathrm{~Hz}$, the spectra is valid in a maximum range from $0.1 \mathrm{~Hz}$ to $48 \mathrm{kHz}\left(1.2 \cdot 10^{-5}<\operatorname{Sr}_{D}<6\right)$.

The high-speed Schlieren image sequence of the recompression shock is additionally processed by determining the standard deviation of the gray values of each pixel within the sequence. The result is an image that shows the local deviation of the shock from the mean (Fig. 7). Pixels with a lighter gray have a higher deviation from the mean value than pixels with a darker gray, which is used to extract the mean location of the recompression shock.

\section{$2.2 \quad$ Numerical Approach}

The Navier-Stokes equations for compressible flows are solved in conservative form with a mixed central-upwind AUSM (advective upstream splitting method) scheme of second-order accuracy with low dissipation [11]. After splitting the inviscid fluxes into a convective and a pressure part and inserting the local speed 
of sound $c$, the convective part is reformulated using a Mach number weighted interpolation

$$
F^{c}=\frac{1}{2}\left[\left(\mathrm{Ma}_{+}+\mathrm{Ma}_{-}\right)\left(f_{-}^{c}+f_{+}^{c}\right)+\left|\mathrm{Ma}_{+}+\mathrm{Ma}_{-}\right|\left(f_{-}^{c}-f_{+}^{c}\right)\right] .
$$

The fluxes $f_{ \pm}^{c}$ and the Mach number $\mathrm{Ma}_{ \pm}$at the cell boundaries are determined by left and right interpolated variables obtained using the MUSCL approach according to van Leer [12]. The viscous terms are discretized by a central scheme of second-order accuracy. The temporal integration is performed by an explicit 5 -stage Runge-Kutta scheme of second-order accuracy, optimized for maximum stability of a central scheme. For the calculation of the base area, a LES is performed, following the monotone integrated LES (MILES) approach [13]. No explicit subgrid scale (SGS) model is implemented, but the dissipation of the numerical scheme serves as an implicit SGS model [14]. The shock treatment in the scheme is accounted for by high spatial resolution in the vicinity of the shocks without reducing the overall second-order accuracy [15].

The flow around the main body is simulated using the one-equation RANS model according to Spalart and Allmaras [16]. The results of the RANS solution close to the trailing edge of the main body are used initially as inflow conditions for the base flow domain. The turbulent viscosity of the RANS model is used to generate physical turbulent fluctuations at the inlet of the LES domain [17, 18]. The Reynolds stress is reconstructed starting from a normalized stochastic velocity signal, which is disturbed by a superimposition of turbulent structures with prescribed geometrical shape and random signs and position. A body force is added to the wall-normal momentum equation on a number of control planes at different streamwise positions in order to match the turbulent flow properties of the LES with the given RANS values $[19,20]$. To keep the transition zone between the RANS and LES domains small, the added synthetic turbulence accords to Jarrin et al. [21].

The geometry is reproduced by a structured multiblock grid (Fig. 8), which is split into two sections. One domain covers the main rocket geometry, in which the fully three-dimensional RANS simulation is carried out and the second encompasses the sting support, thus capturing the highly time-dependent base area and the wake. The 360 degree grid extends to physical values of $2.5 \mathrm{D}$ downstream of the base shoulder in the streamwise direction and captures the oblique shock in the radial direction. The zonal overlapping zone spans up to 1 diameter upstream from the base shoulder. The grid possesses a maximum resolution of $\left(\Delta x_{\text {wall }}^{+}, \Delta r_{\text {wall }}^{+}, \Delta \Phi_{\text {wall }}^{+}\right)=(500,1,150)$ within the RANS domain and of $\left(\Delta x_{\text {wall }}^{+}, \Delta r_{\text {wall }}^{+}, \Delta \Phi_{\text {wall }}^{+}\right)=(50,1,40)$ within the LES domain with $x$ being the streamwise, $r$ the wall-normal, $\Phi$ the spanwise coordinate and the wall unit equal to $1.5 \cdot 10^{-4} D$ based on the main body diameter. Additional refinement is realized along the separating boundary layer and the free shear layer. The 


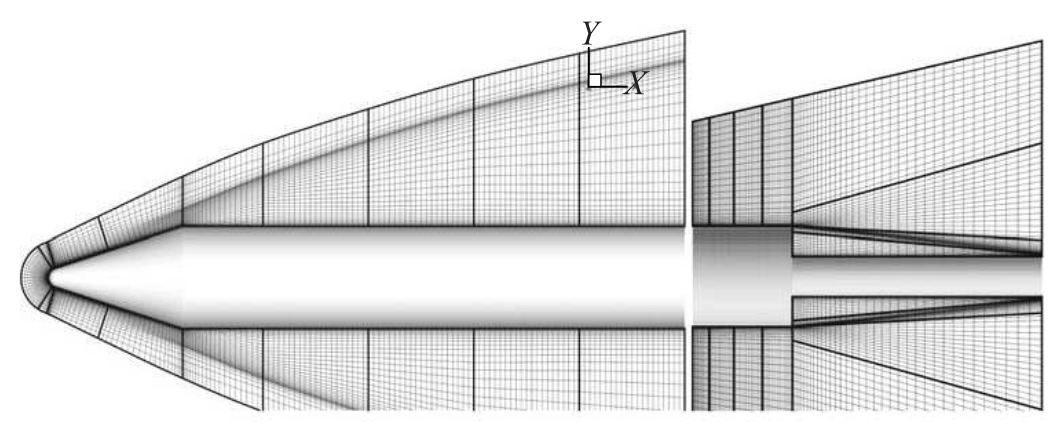

Figure 8 Computational grid for the sting support configuration

$16.2 \cdot 10^{6}$ grid points of the LES domain are equally distributed over 16 blocks to allow load-balancing for an efficient parallel computation.

The boundary conditions are prescribed as follows. Following a characteristics approach, at supersonic boundaries, all variables are prescribed or extrapolated, respectively. At solid walls an isothermal no-slip condition is imposed, since the numerical results are compared to short time wind tunnel tests that lead to a negligible heating of the model.

Analogously to the experimental data analysis the numerical spectral analysis of unsteady pressure fluctuations on the outer surface and recompression shock movements are performed using the fast Fourier transform algorithm. The basis of the spectral analysis is formed by 1024 data samples with a constant time step of $3 \cdot 10^{-5} \mathrm{~s}$ for the Hanning window function. In accordance with the NyquistShannon sampling theorem, the maximum resolved frequency is $16,000 \mathrm{~Hz}$ or $\mathrm{Sr}_{D}=2$, while the minimum resolved frequency is limited by the physical simulation time to $24 \mathrm{~Hz}$ or $\operatorname{Sr}_{D}=0.003$, which reasonably covers the frequency spectra of interest.

\section{RESULTS}

The discussion of the experimental results is divided into a precursory part where the flow upstream of the base is discussed and a main part that only focuses on the base flow. An IR image of the wind tunnel model and the Pitot pressure at the base measured by a Pitot rake is the foundation for the investigations here. In the main part, the mean location of the recompression shock as well as the corresponding spectra is presented. Lastly, data of the pressure fluctuations 
directly on the base are provided by means of spectral analysis for different dummy nozzles.

\subsection{Forebody Measurements}

The underlying idea of the forebody measurements is to deliver representative parameters as input parameters for numerical studies. A first impression can be gained by the qualitative result of the IR-thermography (Fig. 9). It shows the surface temperature of the wind tunnel model color-coded from blue to red, whereas red denotes a higher temperature. Due to the perspective of the camera, the discussion of the cone is neglected. It can be seen that a temperature raise is first located at the tip of the strut and spreads angularly over the surface further downstream. At the base of the model, the temperature transition covers almost the whole cylinder. Nevertheless, lower temperatures can still be detected at the strut-averted side. Evolving from the tip of the strut, the shock and expansion causes confined regions with temperature variations, which extend downstream along the main cylinder.

Figure 10 depicts the Pitot pressure, which is measured at the rear end in radial direction from the model surface at the strut-averted side. For comparison, the Pitot pressures from numerical simulations are plotted in the same graph. Close to the wall between $\Delta r=0.2$ and $3.2 \mathrm{~mm}$, the numerical simulation for the laminar case correlates with the experimental results. However, at higher radial distances, the Pitot pressure measurements tend to follow the curve given by the numerical results for the turbulent case. At about $r=15 \mathrm{~mm}$, the Pitot pressure profile reaches a plateau in experiments as well as in numerics. Slight discrepancies between the two methods can be seen in the final value of the Pitot pressure. Also, the two different runs that are necessary to gather information about the full height of the boundary layer are not completely congruent. No satisfying explanation is found for this deviation.

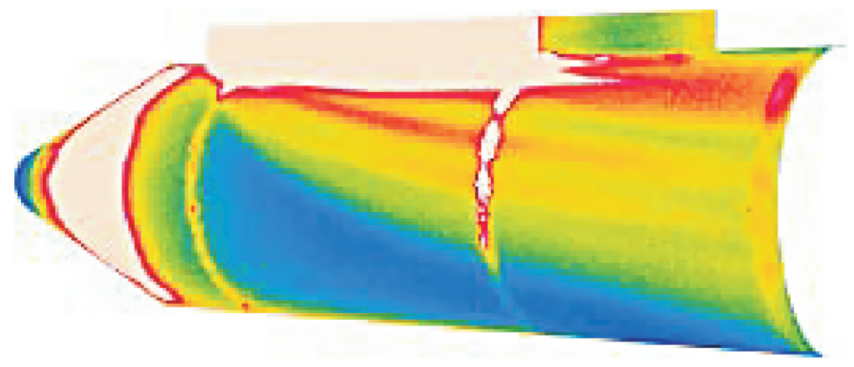

Figure 9 Infrared image showing the surface temperature of the forebody for the investigation of the boundary layer transition. 


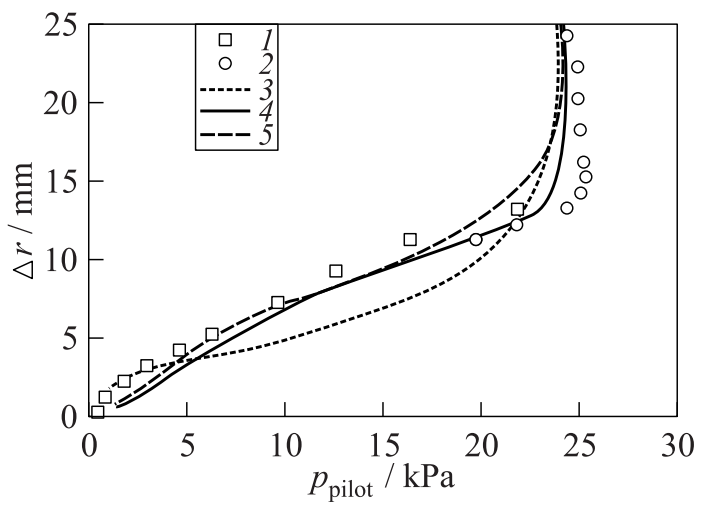

Figure 10 Experimental $\left(\Phi=180^{\circ}\right)(1-$ experiment 1 and 2 - experiment 2$)$ and numerical Pitot pressure of the boundary layer. Numerical Pitot profiles reflect a laminar boundary layer (3) and two turbulent boundary layers based on the turbulence models Spalart-Allmaras (4) and Wilcox $k-\omega$ (5)

\subsection{Base Flow Measurements}

One of the most striking features of the base flow is manifested in the recompression shock. After the expansion at the rear end, the flow deviates towards the axis and causes a recompression region downstream of the base and consequently leads to the recompression shock. This is measured with the high-speed Schlieren method and analyzed as described before concerning the mean location of the shock and plotted in Fig. 11 in $x-r$ coordinates. The continuous lines represent the investigated dummy nozzle configurations and the base is indicated along the $r$-axis as a thick black line. The recompression shock locations are plotted

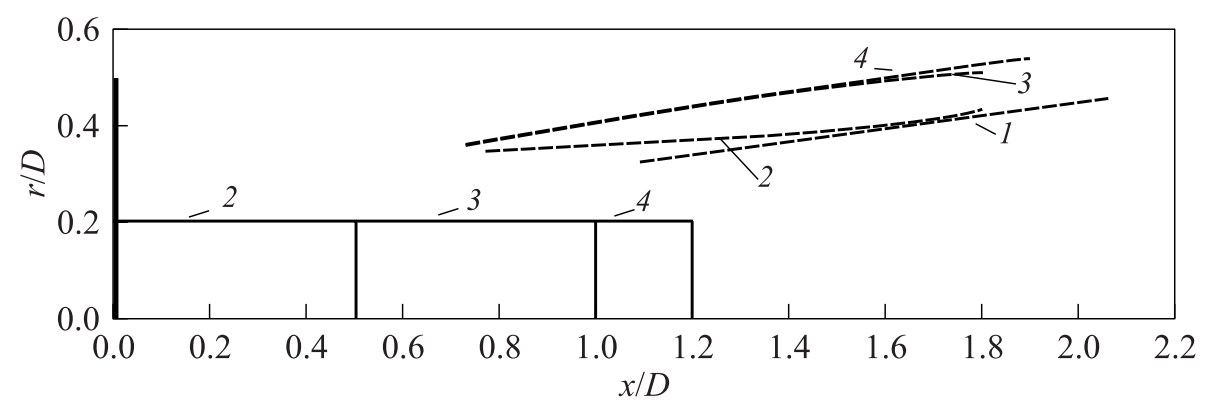

Figure 11 High-speed Schlieren experimental results concerning the mean recompression shock location for different nozzle lengths $L$ at the strut-averted side $\left(\Phi=180^{\circ}\right)$ : $1-L / D=0 ; 2-0.5 ; 3-1.0$; and $4-L / D=1.2$ 


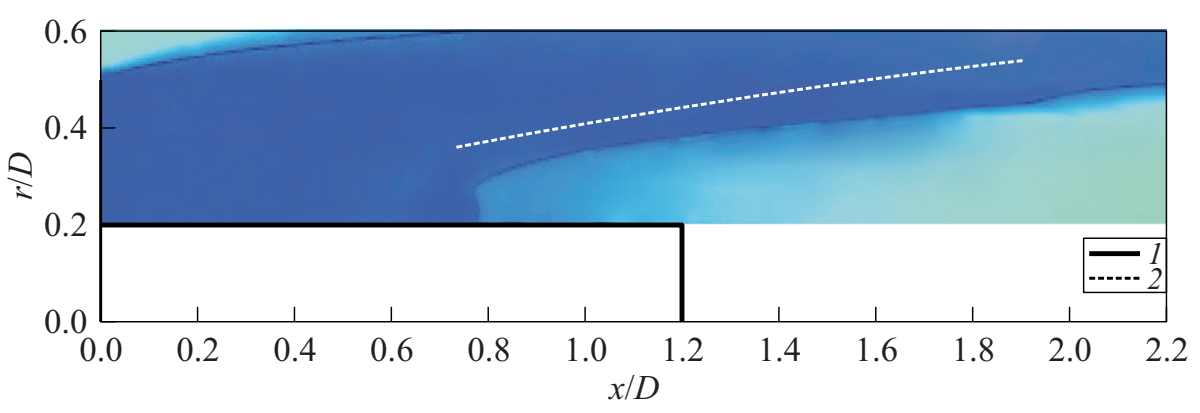

Figure 12 Numerical result concerning the mean recompression shock location (1, $L / D=\infty)$ in comparison to the experimental results for a dummy nozzle of $L / D$ $=1.2(2)$.

as dashed lines. In Fig. 11, a sketch of the dummy nozzle and the recompression shock location are plotted.

For the case with no dummy nozzle, the fine line of the recompression shock can first be detected at about 1.1 diameter lengths downstream of the base at $r / D=0.325$. The shock angle is $7.6^{\circ}$. The most outstanding observation after a applying a dummy nozzle with a length of $L / D=0.5$ refers to the kink of the shock at $x / D=1.42$ and $r / D=0.38$. This indicates a shock interaction and will be discussed in section 4 . Further upstream to that kink, the shock angle is $2.9^{\circ}$. Downstream to that kink, the shock location coincides with the shock location of the configuration without a nozzle. A lengthening of the dummy nozzle to 1.0 causes a parallel shift of the shock location of 0.6 diameters upstream to the base. A further lengthening to 1.2 does not affect the shock location any more. The shock location of the two longest dummy nozzles coincide.

The corresponding numerical investigations differ from the experimental result. Figure 12 shows the pressure in grey codes in the same coordinate system as the graph in Fig. 11. The darker grey indicates a lower pressure. The experimental result for the recompression shock of the nozzle with $L / D=1.2$ is plotted along with the numerical data. It can be seen by the pressure increase that the shock is located approximately 0.4 diameters further downstream in comparison to the experimental shock location result. The shock angle shows a good match with the experimental results.

The high-speed Schlieren video reveals an oscillation of the recompression shock around its mean value. The instantaneous vertical position of the shock is extracted and subjected to its amplitude spectra. Figure 13 shows the vertical amplitude in pixels over the Strouhal number. An arbitrary vertical location of the recompression shock is chosen since the spectra have shown to be independent of the location. The various curves (1-4) represent the different dummy nozzle lengths. A polynomial of the order 8 is fitted to the curves to give a better 


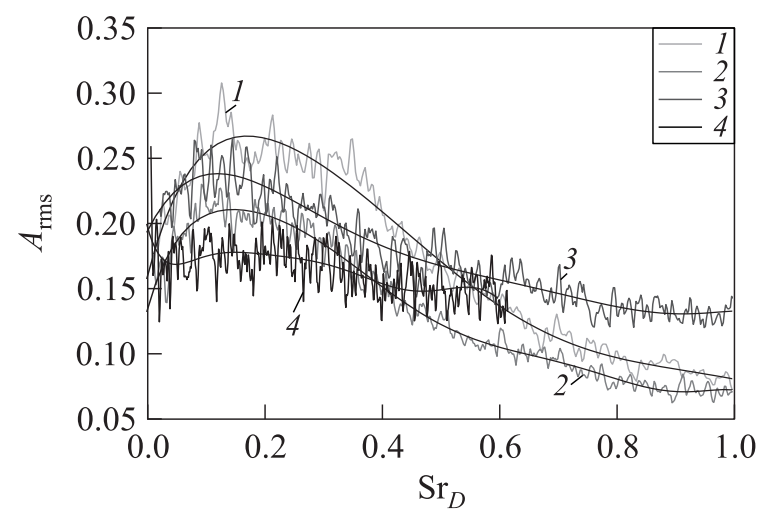

Figure 13 Experimental spectra of the recompression shock location for different dummy nozzle lengths: $1-L / D=0 ; 2-0.5 ; 3-1.0$; and $4-L / D=1.2$

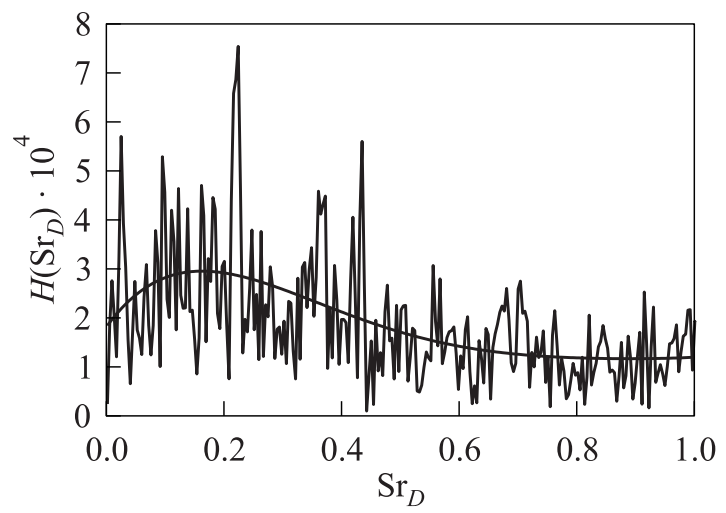

Figure 14 Numerical spectra of the recompression shock location calculated

impression of the course. It can be seen that the peak level for all dummy nozzles centers at about $\operatorname{Sr}_{D}=0.18-0.19$ independently of the dummy nozzle. Figure 14 also depicts the spectra of the recompression shock and a polynomial of the order 5, but from numerical simulations. Although only 1024 samples are used here, the polynomial shows clearly a tendency to center around $\operatorname{Sr}_{D}$ $=0.18$. The numerical spectra features additional peaks at higher frequencies at about $\operatorname{Sr}_{D}=0.37$ and 0.42 , which do not show in the experimental spectra. No explanation is found for that difference yet.

The information of the pressure transducers on the base are used to gain further insight into the unsteady nature of the recirculation zone. The pressure spectra for various positions on the base are given in Figs. 15 and 16, whereas 
the first one delivers a detailed view on the dominant Strouhal numbers and the latter intends to give a spacial impression of the pressure fluctuations on the base. Figure 16 contains the data of Fig. 15.

The pressure spectrum at three outer azimuthal locations $(\Phi$ $=180^{\circ}, 270^{\circ}$, and $\left.315^{\circ}\right)$ with about the same radial distance $(r=40$ and $45 \mathrm{~mm}$ ) for the configuration without a dummy nozzle is given in Fig. 15. Independently of the azimuthal location, the three pressure transducer feature a dominant Strouhal number at about $\mathrm{Sr}_{D}$ $=0.21-0.22$ and some pressure fluctuations seem to oscillate with a broader frequency, which centers around 0.31-0.33. Additional nondimensional frequencies can be detected at $\mathrm{Sr}_{D}=0.08-0.09(\Phi$ $=180^{\circ}$ and $315^{\circ}$ ) and a strong signal at $\operatorname{Sr}_{D}=0.145 \quad(\Phi$ $=180^{\circ}$ and $270^{\circ}$ ). These graphs are chosen to show exemplarily the reoccurring nondimensional frequencies of $\operatorname{Sr}_{D}=0.08-0.09,0.145$, $0.21-0.22$, and about $0.31-0.3$. An interpretation with respect to the underlying physical effects is given in section 4.

As an additional information to the previous graphs, Fig. 16a to Fig. $16 d$ depict the radial distance according to the pressure transducer arrangement introduced in Fig. 6 for $\Phi=180^{\circ}, 225^{\circ}, 270^{\circ}$, and $315^{\circ}$. Taking all four graphs into consideration, it can be observed that the level of the pressure fluctuations increases continuously towards the center of the base and oscillate with a dominant frequency of $\mathrm{Sr}_{D}=0.08$. Towards the outer region, a depen-

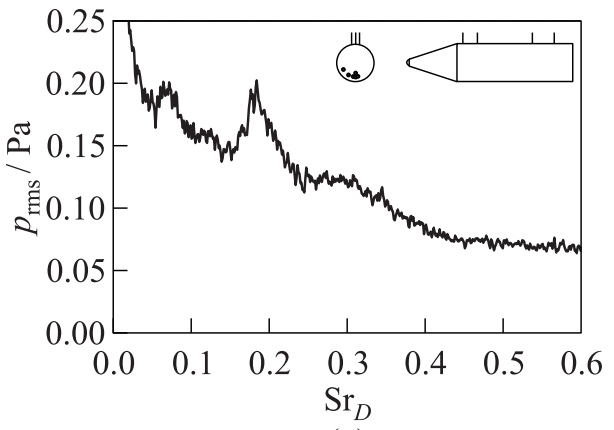

(a)

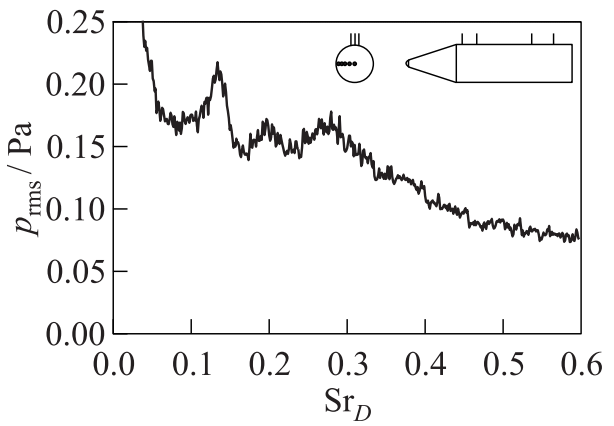

(b)

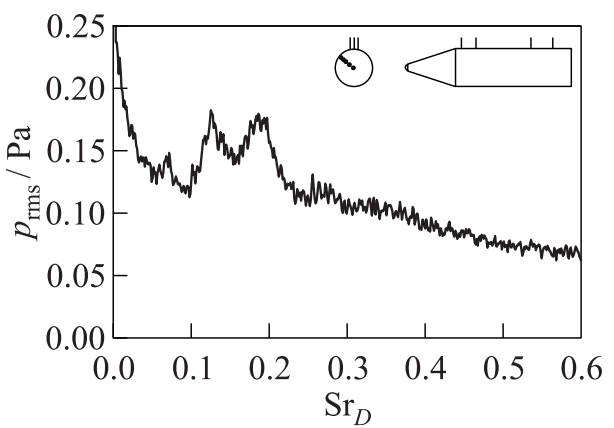

(c)

Figure 15 Experimental pressure spectra at different azimuthal locations without a dummy nozzle: (a) $r=40 \mathrm{~mm}$ and $\Phi=190^{\circ}$; (b) $r=45 \mathrm{~mm}$ and $\Phi=270^{\circ}$; and $(c) r$ $=45 \mathrm{~mm}$ and $\Phi=315^{\circ}$ 


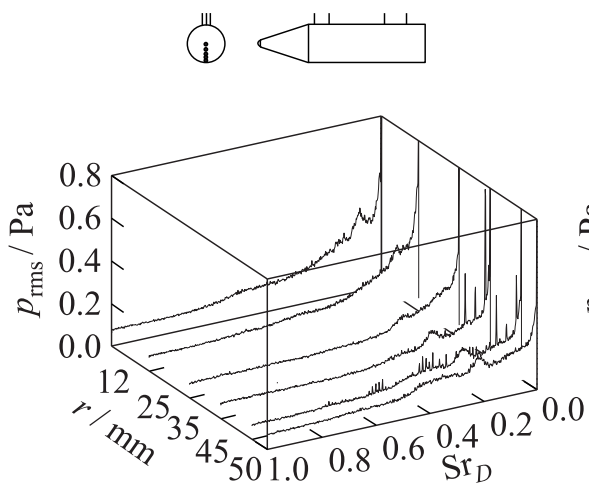

(a)

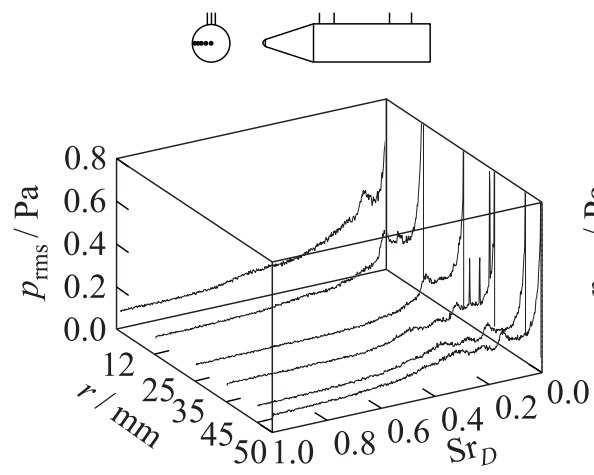

(c)

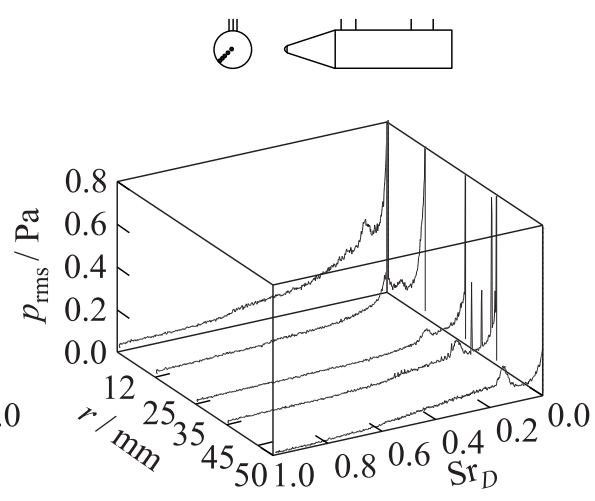

(b)

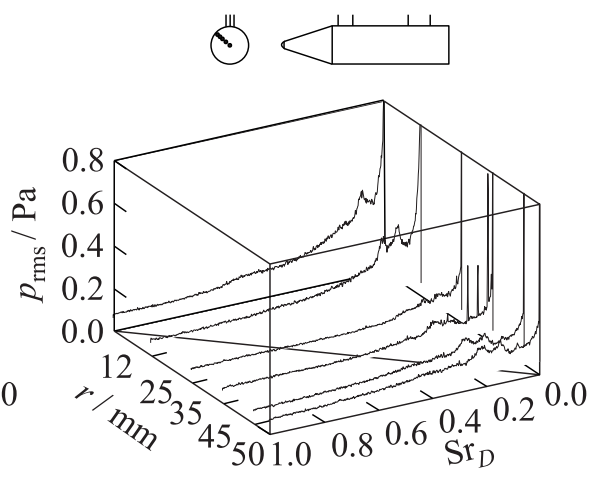

(d)

Figure 16 Pressure spectra for radial pressure transducer alignment at different angles $\Phi$ : (a) $180^{\circ}$; (b) $225^{\circ} ;(c) 270^{\circ}$; and $(d) 315^{\circ}$

dency to the azimuthal location can be detected. Whereas the same nondimensional frequency is detected for the transducers at $r=12$ and $25 \mathrm{~mm}$, it can be denoted that additional frequencies appear in the pressure spectrum. For example, the transducers at $\Phi=225^{\circ}, 270^{\circ}$, and $315^{\circ}$ measure fluctuations with a Strouhal number of about $\mathrm{Sr}_{D}=0.145$. This frequency is also part of the spectra for the outer sensors at $r=35,45$, and $50 \mathrm{~mm}$ at the same azimuthal location. Even more dominant nondimensional frequencies join the pressure spectra when approaching the outer region of the base. As it is discussed in the detailed examination of Fig. 15, the outer transducer exhibit additional frequencies at about $\mathrm{Sr}_{D}=0.21-0.22$ and a broad-band signal centering at about $0.31-0.33$ in the pressure spectra. Meanwhile, the fluctuations at the center Strouhal number of 0.08 almost completely disappear. A note concerning the distinct peaks of 


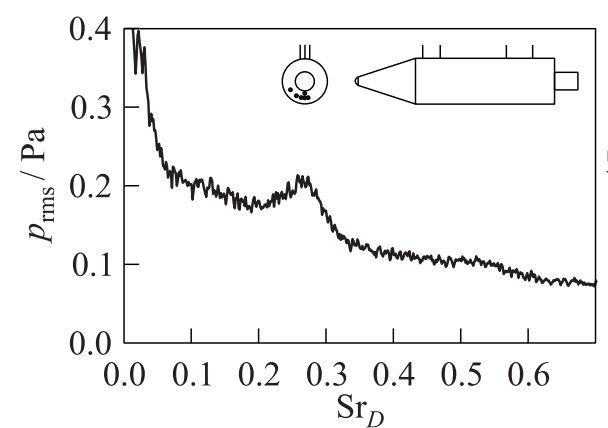

(a)

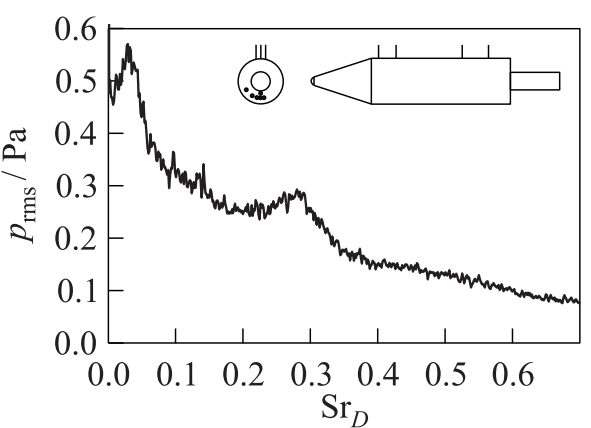

(b)

Figure 17 Experimental pressure spectra for different nozzle lengths at $r=40 \mathrm{~mm}$ and $\Phi=190^{\circ}:$ (a) $L / D=0.5 ;$ and $(b) L / D=1.0$

the transducers at $r=35$ and $45 \mathrm{~mm}$ : these peaks are most likely caused by electromagnetic interferences since they are independent of experimental input conditions.

The influence of a nozzle attached to the end of the generic launcher on the pressure fluctuations is discussed in the following. The data is presented in the same manner as before. A single pressure spectrum is extracted from the graphs that show the spacial distribution and is then discussed in detail.

Figures $15 a, 17 a$, and $17 b$ show the pressure spectra at $r=40 \mathrm{~mm}$ and $\Phi=190^{\circ}$ for the configuration without a nozzle, and with a dummy nozzle of the length $L / D=0.5$ and 1.0 , respectively. It can clearly be seen that the pressure spectrum undergoes a crucial change. For the configuration without a dummy nozzle, several dominant Strouhal number are identified at about 0.08, $0.21-0.22$, and $0.31-0.33$. The pressure spectrum for both dummy nozzles are very similar. It features a peak at about $0.025-0.03$ and another distinct Strouhal number at about 0.27 .

The pressure fluctuations from numerical simulations for configuration $B$ (Fig. 18) are also extracted at the corresponding location at $r=40 \mathrm{~mm}$. A least squares 10th-order polynomial is fitted through the spectra in order to receive the trend. As it can be seen, the course of the polynomial features a peak at a low nondimensional frequency of about $\mathrm{Sr}_{D}=0.03$. Since the physical time of the performed LES is given by 1024 samples with a constant time step of $3.1 \cdot 10^{-5} \mathrm{~s}$ the lowest resolved frequency is $\operatorname{Sr}_{D}=0.003$ or $f=24 \mathrm{~Hz}$. The dominant peak at $\mathrm{Sr}_{D}=0.024$ or $f=200 \mathrm{~Hz}$ is covered by approximately 8 wave lengths, which is satisfactory for a reliable detection of the dominant frequency. A secondary peak of small amplitude around the Strouhal number of 0.27 is visible for the used polynomial of higher order, whereas the peak is not as distinct as in the experimental data presented in Fig. 17b. This agrees with the 


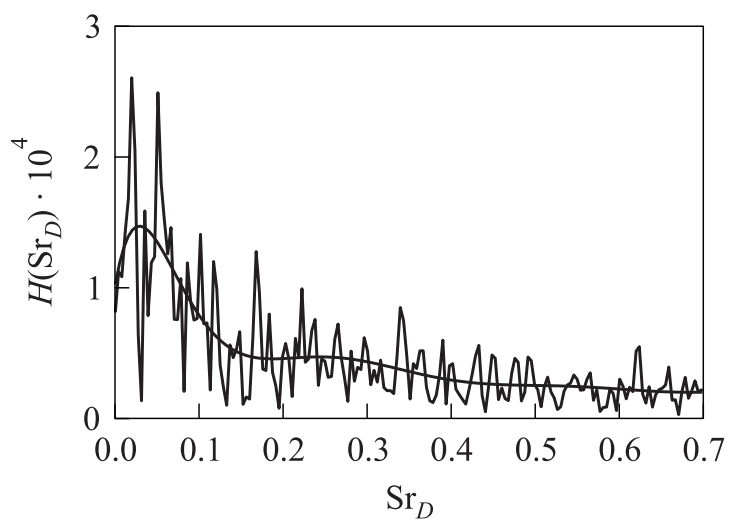

Figure 18 Numerical pressure spectra determined at the position $r=40 \mathrm{~mm}$

tendency towards smaller amplitude of the broad-band fluctuations at $\mathrm{Sr}$ about 0.27 with the increasing nozzle length shown in Fig. $17 b$, for the numerically investigated configuration represents an endless dummy nozzle extension. Overall, the course of the polynomial resembles the course of the pressure spectra found in experiments.

To provide insight into the dominant effects of the observed dynamic behavior, the numerical results are analyzed considering low-frequency phenomena in the wake region. A random time period corresponding to an observed dominant frequency is extracted from the LES data. Subsequently, the period is divided into several equal time segments each of which was time-averaged over its length to filter out the small resolved turbulent scales fluctuations for better visualization and determination of the large scale fluid motion. The obtained sequence of the filtered flow field states is analyzed considering periodical flow phenomena.

Corresponding to the first dominant mode in the pressure spectrum at $\operatorname{Sr}_{D}$ $=0.024$ or $200 \mathrm{~Hz}$ shown in Fig. 18, a random time period of $5 \mathrm{~ms}$ is extracted from the LES data. This period is divided into 5 equal time segments each of which is time-averaged over $1 \mathrm{~ms}$. The streamlines and pressure fields of these 1-millisecond time-averaged samples are presented in Fig. 19. The recirculation zone is represented by two counter rotating and interacting toroidal vortices whose dimensions and core positions change in time. A comparison of the temporal states in Fig. 19 reveals that the dominant mode in the pressure spectrum at $\mathrm{Sr}_{D}=0.024$ corresponds to the movement of the core of the toroidal vortex 1 near the inner base corner. During one period of $5 \mathrm{~ms}$, the core 1 completes a full clockwise rotation around its center axis which results in the observed mode in the pressure spectrum. The core position of the second toroidal vortex 2 oscillates only in the radial direction. However, the frequency is doubled and 


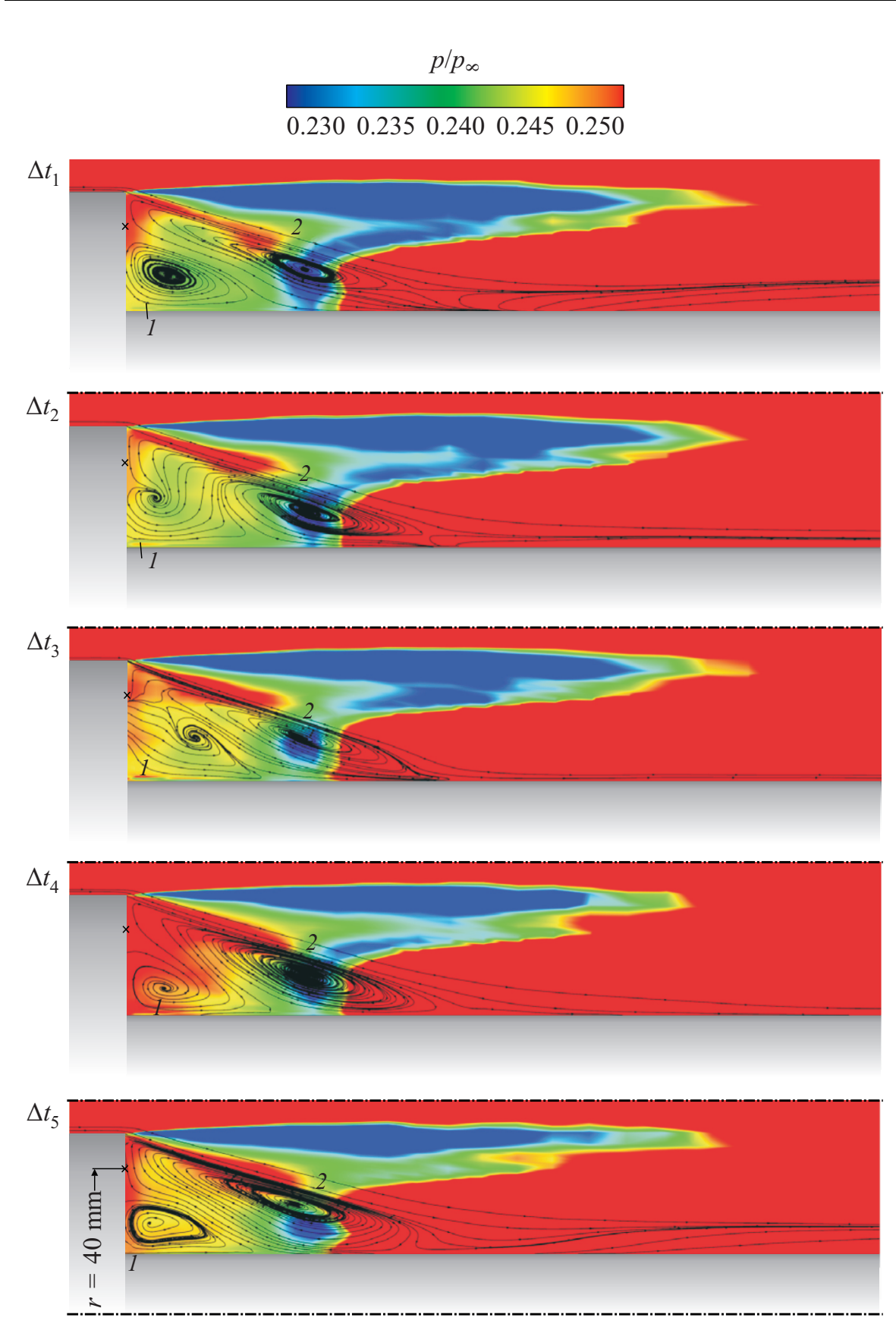

Figure 19 Filtered temporally intermediate streamlines and pressure fields of the analyzed time period of $T=5 \mathrm{~ms}$ with $\Delta t_{i}=1 \mathrm{~ms}$. 


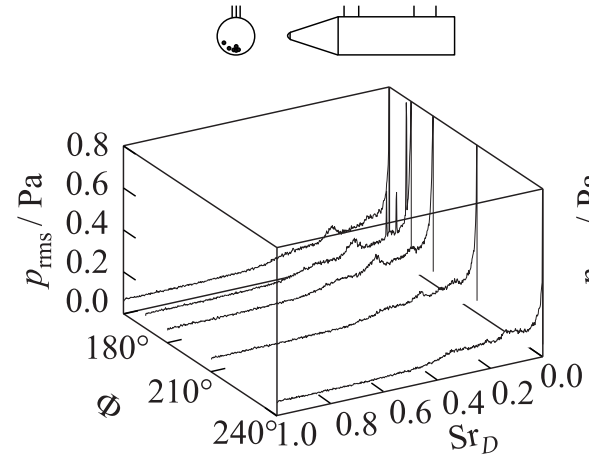

(a)

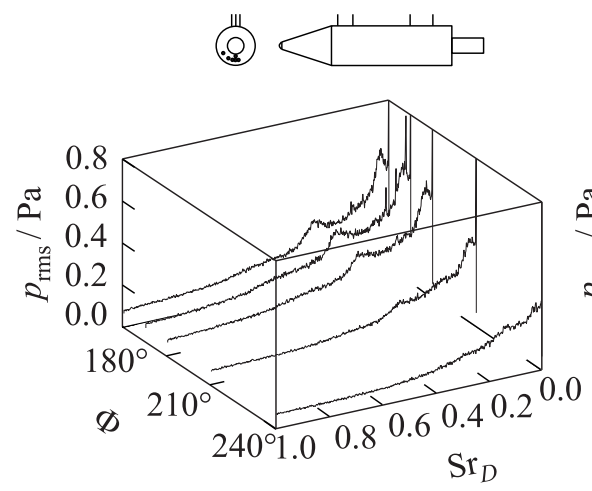

(c)

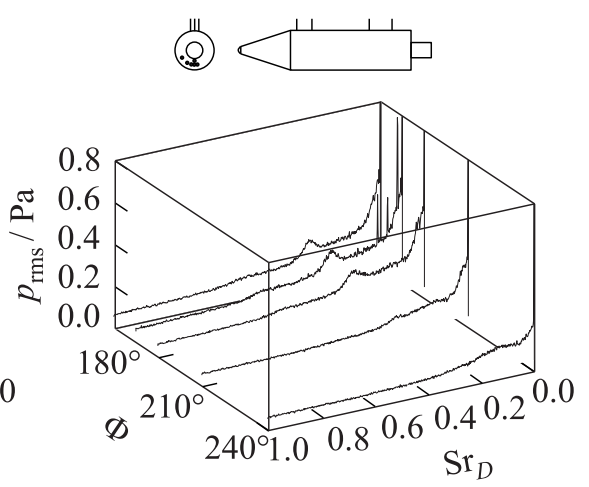

(b)

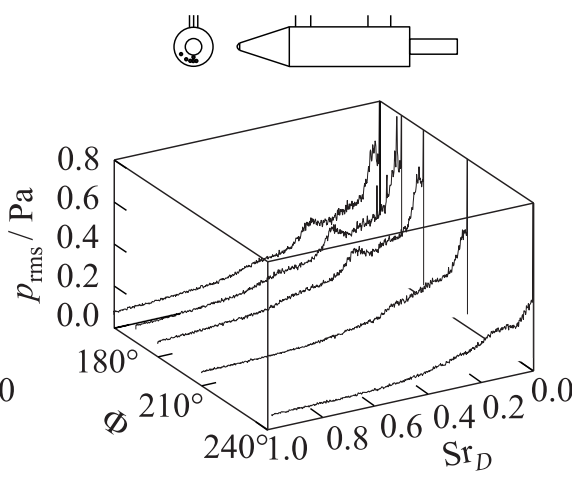

(d)

Figure 20 Experimental pressure spectra for different dummy nozzle lengths at $r=40 \mathrm{~mm}:(a)$ no dummy nozzle; $(b) L / D=0.5 ;(c) 1.0 ;$ and $(d) L / D=1.2$

the amplitude is smaller than that of vortex 1 which corresponds to the second weaker peak at $\mathrm{Sr}_{D}=0.05$ in Fig. 18 .

The spacial distribution of the pressure fluctuations when a nozzle is added to the base is given in Fig. 20 for the transducer arrangement described in Fig. 6 . The spacial direction in the graphs here represent the azimuthal position $\Phi$ at $r=40 \mathrm{~mm}$. Figure $20 a$ contains data of the already discussed pressure spectrum in Fig. $15 a$ with distinct pressure fluctuations Strouhal numbers at about $0.08-0.09,0.21-0.22$, and $0.31-0.33$. The transducers in the clockwise direction towards the strut show mainly the same Strouhal numbers except that frequency contents especially at $0.21-0.22$ get damped.

The short dummy nozzle with $L / D=0.5$ now results in a change in the dominant Strouhal number to $\operatorname{Sr}_{D}=0.27$ (see Figs. $15 a$ and 20b) and some high 
pressure fluctuations at low frequencies with no distinct peak. Other frequency contents can not be detected anymore. The nondimensional frequency of 0.27 is most distinct on the opposite side of the strut, and, as was seen before in Fig. $20 a$, smears out to a broadband signal for the transducers closer to the strut. Along with the frequency shift comes an increase of the pressure fluctuation level.

A lengthening of the dummy nozzle to $L / D=1.0$ (Fig. 20c) causes an overall increase of the pressure level, fluctuations at the distinct Strouhal number 0.27 and a broader signal for the transducers in the clockwise direction towards the strut. Additionally, the low frequency fluctuations now feature a distinct peak at about $0.025-0.03$. A further lengthening of the dummy nozzle to $L / D=1.2$ does not influence the pressure spectra on the base anymore. The courses of the spectra in Figs. $20 c$ and $20 d$ are practically identical. All graphs in Fig. 20 have in common that the pressure spectra feature a symmetric behavior: the course of the graphs for $\Phi=170^{\circ}$ and $190^{\circ}$ essentially coincide.

\section{DISCUSSION}

The boundary layer is considered as one of the main parameters from upstream influencing the recirculation zone. Hence, a brief discussion is devoted to the incoming boundary layer before focussing on the interpretation of the unsteady effects in the recirculation zone and the crucial differences between a free reattachment of the flow in comparison to a reattachment on a solid surface.

The measurements results of the Pitot pressure (see Fig. 10) and the infrared image (see Fig. 9) upstream of the base both indicate that the boundary layer is not fully turbulent. Close to the wall, the Pitot pressure coincides with the calculated laminar course. With an increasing radial distance, the Pitot pressure measurements approach the course for the calculated turbulent boundary layer. A laminar-turbulent transition of the boundary layer is connected with a temperature increase on the surface. The infrared images show that the tip of the strut is triggering a temperature raise on the strut-facing side. For the strut-averted side, it can not clearly be determined if the transition takes place.

Despite the ambiguous information from the incoming boundary along the main body, the investigated case shows strong similarities to the case described in [10] with a turbulent boundary layer and a Reynolds number of $16 \cdot 10^{6} \mathrm{~m}^{-1}$ and comparisons will be made later in this section.

The pressure spectra reveal that the internal flow dynamics and the flow topology of the recirculation zone undergoes a crucial change when a nozzle is attached to the base region. In contrast to that, the recompression shock oscillates with the same periodicity. Figure 11 shows that the recompression shock is shifted parallelly upstream to the base when a nozzle is attached, while the oscillation of this shock takes place with the same nondimensional frequency of 
about $0.18-0.19$ (see Fig. 13). A periodicity in this range is also seen in [10] for a higher unit Reynolds number of $16 \cdot 10^{6} \mathrm{~m}^{-1}$ with the same wind tunnel model and various nozzle diameters. The numerical simulations calculated the recompression shock to be further downstream than measured in experiments (see Fig. 12). This seems to be caused by the different support configurations. Independent to the shock location and the extension of the recirculation region, the polynomial through the spectra of the shock location returns a dominant frequency at the same Strouhal number of 0.19. The results indicate that the dynamic behavior of the recompression shock is not a strong function of the Reynolds number, the geometry in the base region or the extension of the recirculation region since a Strouhal number of 0.18 to 0.19 can be found in all of these cases. In literature, a Strouhal number of about 0.2 is often related to the flapping motion of the von Kármán vortex street caused by the unsteady separation of the flow from a blunt body.

A direct link between the dynamic behavior of the recompression shock and the recirculation region is difficult. For the free reattachment case, for example, a number of different distinct peaks can be detected in the pressure spectra (see Figs. $16 a$ to $16 d$ ) indicating more than just one mode. Dominant Strouhal numbers are detected at $0.08-0.09,0.145,0.21-0.2$ and at between 0.31 and 0.33 . An extrapolation of the pressure fluctuations at a few measurement locations is not feasible. In literature, fluctuations with a periodicity of $0.08-0.09$, for example, are often related to an axisymmetric growth and decay process of the recirculation zone and, as mentioned before, the periodicity of 0.2 is connected to the von Kármán vortex shedding. More research has to be done to identify modes that oscillate with the Strouhal numbers of 0.145 and at about 0.32 . A common feature that can be observed in all spectra concerns the low pressure fluctuations close to the edge of the base, which intensify towards the center of the base where it reaches a maximum peak. This effect might be due to the focussing pressure fluctuations emanating circumferentially from the shear layer. Further, it can be seen that there is an azimuthal dependency of the pressure fluctuations, which give rise to the idea of azimuthal pressure modes seen in [22]. On the other hand, it can not be stated without doubt that a preferred orientation is introduced by the strut since the transducers $10^{\circ}$ left and right from the strut-averted side are comparable.

No direct correlation between the recompression spectra and the basepressure spectra can be deduced for the reattachment on a solid wall either. Additionally, the base-pressure spectra itself reveals crucial differences if attached freely or on a solid wall. The pressure spectra of the two different configurations differ in the number of distinct peaks, the nondimensional dominant frequencies and the level of the pressure fluctuations.

For the dummy nozzle of $L / D=1.0$ and 1.2, Fig. 11 shows an identical location for the recompression shock. This suggests that the recirculation region is identical as well and, hence the same pressure spectra should be an outcome 
of this finding. This can be seen in the striking similarity of Figs. $20 c$ and $20 d$. The flow reattaches for both cases on a solid wall and simplifies the flow dynamic in a sense that only two dominant Strouhal number can be detected at about 0.025-0.03 and 0.27. An explanation to the reduction of the amount of distinct frequencies might be that the application of the dummy nozzle influences the recirculation zone in such a way that only symmetric effects like the growth and decay of the recirculation region or the varicose mode shedding from the shoulder play a role in the dynamics. It seems obvious that antisymmetric movements are somehow suppressed.

The numerical investigations on the comparable configuration $B$ reaveal peaks in the pressure spectra at $\mathrm{Sr}_{D}=0.024$, which agrees reasonably with the experimental findings at 0.025-0.03. Further, Fig. 19 showed that this peak results from the radial and axial movement of the toroidal corner vortex. A second mode with a frequency twice as high is assigned to the movement of the larger vortex. This mode is not found in experiments.

A special case is considered for the dummy nozzle of $L / D=0.5$. The fact the recompression shock does not coincide with the shocks of the longer nozzles (see Fig. 11) means that the flow is not completely reattached. Yet, a recompression with a realignement of the flow takes place on the dummy nozzle, where the flow is deviated in the axisymmetric direction. This is where a first shock is formed. At the rear end of the nozzle, the flow is deviated towards the axis again and must finally undergo another recompression, where further recompression waves emanate. The two shocks interact at the position where the kink is located. The angle of the first shock is lower since the reattachment or realignment on the nozzle is most likely not complete. Consequently, the recirculation region is not identical to the one with the longer nozzles and different pressure spectra can be observed (see Fig. 20b). The dominant Strouhal number of 0.27 can also be found. But there is no distinct low frequency fluctuation at $0.025-0.03$ and the overall pressure level is lower.

The investigations at hand reveal striking similarities to reference [10], which are carried out on the same model at a unit Reynolds number $16 \cdot 10^{6} \mathrm{~m}^{-1}$ and at Mach 6.0. For the free reattachement of the flow, comparable features like the maximum in the pressure fluctuations at $\mathrm{Sr}_{D}=0.08$ in the center of the base can be found as well. Closer to the edge of the base, the former experiments also show a distinct peak at Strouhal number 0.2. In contrast to the experiments here, no peaks are found at $\mathrm{Sr}_{D}=0.145$ and at between 0.31 and 0.33 . The former experiments reaveal a peak at 0.38 instead. The fluctuations of the recompression shock are identical. This indicates that in this flow regime the recompression shock and the fluctuations that take place at 0.08 and at approximately $0.2 \mathrm{seem}$ to have a small dependency on the Reynolds number and the incoming state of the boundary layer. Vice versa, this might mean that some fluctuations show a sensitivity to criteria mentioned before. For the $10 \cdot 10^{6} \mathrm{~m}^{-1}$ case, the incoming boundary layer or Reynolds number seems to be the reason for fluctuations at 
0.145 and at broadband signal at about 0.32 for the free reattachment of the flow.

The reattachement of the flow on a solid wall for $\operatorname{Re}_{U}=16 \cdot 10^{6} \mathrm{~m}^{-1}$ follows the same pattern as for the current investigations. In both cases, a distinct peak at $\mathrm{Sr}_{D}=0.27$ can be observed and the recompression shock oscillates in the vertical direction with a Strouhal number of 0.2. The mean recompression is shifted closer to the axis and the shock angle is steeper than from the case with $\operatorname{Re}_{U}=10 \cdot 10^{6} \mathrm{~m}^{-1}$. Consequently, the deviation of the flow from the shoulder is even larger there.

\section{CONCLUDING REMARKS}

In the present paper, axisymmetric base flows at $\mathrm{Ma}=6.0$ at a Reynolds number of $10 \cdot 10^{6} \mathrm{~m}^{-1}$ are investigated experimentally for different dummy nozzles and compared to the numerical results from the zonal RANS/LES method applied on the geometry with a sting support. Measurements on the forebody by means of a Pitot rake and thermography are conducted to provide information about the boundary layer at the rear end of the main body. The base flow is studied by measuring the unsteady base-pressure fluctuations complemented with the data from unsteady high-speed Schlieren measurements. At the corresponding locations, measurement data and results from the numerical simulations are compared to each other.

On the one hand, the measurement reveal that no direct correlation can be drawn between the recompression shock oscillation and the pressure fluctuations of the recirculation region. The recompression shock oscillates independently with a Strouhal number of $\operatorname{Sr}_{D}=0.18-0.19$ without or with dummy nozzles. On the other hand, it is also shown that the dynamics of the recirculation region is heavily influenced by the reattachment of the flow. For a free reattachment, dominant Strouhal number can be measured at $0.08-0.09,0.145,0.21-0.22$ and at about 0.32 . For the blunt base configuration, the pressure fluctuations focus to a peak pressure level at $0.08-0.09$ in the center of the base. For a reattachment on a solid surface, the pressure fluctuations are suppressed or shifted to lower frequencies. Peaks can be seen at $0.025-0.03$ and 0.27 .

The applied zonal RANS/LES method is demonstrated to predict satisfactorily the intricate wake flow for high Reynolds numbers. Although the mean location for the recompression flow differs from the experimental results, which is most likely caused by the different supports, the dynamic behavior of the flow is reproduced. For the recompression shock, a dominant Strouahl number of 0.18 is calculated and the pressure fluctuations feature a periodicity of 0.024 and 0.27 . The numerical simulations deliver interesting insights into the physical phenomena of the turbulent flow field. For instance, the driving effect for the 
Strouhal number of $\mathrm{Sr}_{D}=0.024$ can be assigned to the combined radial and axial movement of the toroidal corner vortex. As shown in this paper, the numerical investigations can successfully be used to reduce the variety of prospective driving mechanisms concerning the interaction of the external flow and the recirculation region by determining the physical effects which are responsible for the obtained dynamic behavior.

Due to the difficulties of attributing flow phenomena to certain frequencies, future work from the experimental point of view will focus on a higher spacial resolution of the base-pressure fluctuations. Further, the measurement method of particle image velocimetry will be applied to gain more insights into the mean flow topology, the shear layer and the turbulent velocity. In the future, it is planned to run experiments under more realistic conditions by taking a hot jet plume into account.

\section{ACKNOWLEDGMENTS}

Financial support has been provided by the German Research Foundation (Deutsche Forschungsgemeinschaft - DFG) in the framework of the Sonderforschungsbereich Transregio 40. The help and advice of the technical staff of the supersonic and hypersonic department is gratefully acknowledged. A data acquisition system has been kindly provided by the Institute of Aeronautics and Astronautics, RWTH Aachen.

\section{REFERENCES}

1. Rollstin, L. 1987. Measurement of inflight base-pressure on an artillery-fired projectile. AIAA Paper No. 1987-2427.

2. Geurts, E. G. M. 2006. Steady and unsteady pressure measurements on the rear section of various configurations of the Ariane 5 launch vehicle. 6th Symposium (International) on Launcher Technologies. Munich, Germany.

3. David, Ch., and S. Radulovic. 2006. Prediction of buffet loads on the Ariane 5 afterbody. 6th Symposium (International) on Launcher Technologies. Munich, Germany.

4. Depres, D., S. Radulovic, and H. Lambare. 2006. Reduction of unsteady effects in afterbody transonic flows. 6th Symposium (International) on Launcher Technologies. Munich, Germany.

5. Deck, S., R. Thépot, and P. Thorigny. 2007. Zonal detached eddy simulation of flow induced unsteady side-loads over launcher configurations. 2nd European Conference for Aerospace Sciences.

6. Lüdeke, H., and J. B. Calvo. 2008. A fluid structure coupling of the Ariane-5 during start phase by DES. 6th European Symposium on Aerothermodynamics for Space Vehicles. Versailles, France. 
7. Marié, S., S. Deck, and P.E. Weiss. 2010. From pressure fluctuations to dynamic loads on axisymmetric step flows with minimal number of kulites. Comput. Fluids 31(5):747-55.

8. Sippel, M., and A. Herbertz. 2007. System requirements on investigation of base flow/plume interaction. In: RESPACE - key technologies for reusable space systems. Ed. A. Gülhan. Berlin-Heidelberg: Springer-Verlag. 3-19.

9. Ottens, H. B. A., M. I. Gerritsma, and W. J. Bannink. 2001. Computational study of support influence on base flow of a model in supersonic flow. AIAA Paper No. 20012638.

10. Saile, D., A. Gülhan, and A. Henckels. 2011. Investigations on the near-wake region of a generic space launcher geometry. 17th AIAA International Space Planes and Hypersonic Systems and Technologies Conference. Manuscript ID \#947684.

11. Liou, M. S., and C. J. Steffen. 1993. A new flux splitting scheme. J. Comput. Phys. 107:23-39.

12. Van Leer, B. 1979. Towards the ultimate conservative difference Scheme V. A second-order sequel to Godunov's method. J. Comput. Phys. 32:101-36.

13. Fureby, C., and F.F. Grinstein. 2002. Large eddy simulations of high-Reynoldsnumber free and wall-bounded flows. J. Comput. Phys. 181:68-97.

14. Boris, J. P., F.F. Grinstein, E. S. Oran, and R. L. Kolbe. 1992. New insights into large eddy simulation. Fluid Dyn. Res. 10(4-6):199-228.

15. El-Askary, W., W. Schröder, and M. Meinke. 2003. LES of compressible wall bounded flows. AIAA Paper No. 2003-3554.

16. Spalart, P. R., and S. R. Allmaras. 1992. A one-equation turbulence model for aerodynamic flows. AIAA Paper No. 92-0439.

17. König, D., M. Meinke, and W. Schröder. 2010. Embedded LES/RANS boundary in zonal simulations. J. Turbulence 11:1-25.

18. Zhang, Q., W. Schröder, and M. Meinke. 2010. A zonal RANS-LES method to determine the flow over a high-lift configuration. Comput. Fluids 39(7):1241-53.

19. Spille, A., and H.-J. Kaltenbach. 2001. Generation of turbulent inflow data with a prescribed shear-stress profile. 3rd AFSOR Conference on DNS and LES.

20. Keating, A., G. de Prisco, and U. Piomelli. 2006. Interface conditions for hybrid RANS/LES calculation. Int. J. Heat Fluid Flow 27:777-88.

21. Jarrin, N., N. Benhamadouche, S. Laurence, and D. Prosser. 2006. A syntheticeddy-method for generating inflow conditions for large-eddy simulations. J. Heat Fluid Flow 27:585-93.

22. Meiss, J.-H., and W. Schröder. 2007. Large-eddy simulation of a generic space vehicle. In: RESPACE - key technologies for reusable space systems. Ed. A. Gülhan. Berlin-Heidelberg: Springer-Verlag. 21-39. 